\title{
Electronic transient spectroscopy from the deep UV to the NIR: unambiguous disentanglement of complex processes
}

\author{
Eberhard Riedle, Maximilian Bradler, Matthias Wenninger, \\ Christian F. Sailer and Igor Pugliesi
}

Received 5th February 2013, Accepted 11th February 2013

DOI: $10.1039 / \mathrm{c} 3 \mathrm{fd} 00010 \mathrm{a}$

Complex multi-stage relaxation and reaction pathways after the optical excitation of molecules makes the disentanglement of the underlying mechanisms challenging. We present four examples that a new transient spectrometer with excitation fully tunable from the deep UV to the IR and 225 to $1700 \mathrm{~nm}$ probing allows for an analysis with greatly reduced ambiguity. The temporal resolution of about $50 \mathrm{fs}$ allows us to resolve all relevant processes. For each example there is a new twist in the sequence of relaxation steps that had previously been overlooked. In malachite green it appears that the importance of the phenyl twisting has been overemphasized and rather a charge transfer state should be considered. In TINUVIN-P the predicted twisting as the driving motion for the ultrafast IC is confirmed and leads to a resolution of the earlier puzzle that the sub- 5 ps regime shows kinetics deviating from a pure cooling process despite the sub-ps proton transfer cycle. For the bond cleavage of $\mathrm{Ph}_{2} \mathrm{CH}-\mathrm{Cl}$ and $\mathrm{Ph}_{2} \mathrm{CH}-\mathrm{Br}$ the degree of electron transfer within the radical pair can now be determined quantitatively and leads to a profound understanding of the long term cation yield. For the first time coherent wavepacket motion in the photoproducts is reported. Last but not least the measurement of the GSB recovery in the deep UV allows for the surprising result, that even after $S_{2}$ excitation of cyclopentenones the triplet states are reached with near unity probability within a few picoseconds.

\section{Introduction}

Optical spectroscopy in a wide range of varieties is a prime source of microscopic information on molecular systems and processes. Simple continuous wave ultraviolet-visible (UV/vis) absorption allows the identification of the energies of excited electronic states and infrared (IR) measurements render typical vibrational modes that are used to identify the associated motives in the molecule. The

Lehrstuhl für BioMolekulare Optik, Ludwig-Maximilians-Universität München, Oettingenstr. 67, 80538 München, Germany. E-mail: Riedle@physik.uni-muenchen.de; Fax: (+49)089-2180-9202; Tel: (+49)0892180-9210 
two regimes are complementary in many ways and the skilful choice or combination is often needed.

Modern time resolved versions of UV/vis and IR spectroscopy permit the observation of the temporal evolution of the system with well below $100 \mathrm{fs}$ precision. Particularly in the UV/vis practically all relevant processes can now be resolved. The interpretation of the raw measurements does, however, require the identification of unambiguous signatures of the reaction intermediates and the eventual products. This is by far not trivial, as only the spectrum of the reactants is typically well known and possibly that of the product, if it can be isolated in sufficient quantity and purity. Any short lived intermediates can only be characterized by the transient measurement itself and so there is often a "chicken and egg" dilemma that a model of the reaction is needed to assign the observed spectral signatures and these would be of great help to develop the model.

As the structural motives that determine the vibrational frequencies are quite localized, e.g., $\mathrm{N}-\mathrm{H}$ or $\mathrm{C}-\mathrm{C}$ stretches or specific bending motions in the fingerprint region, at least first guesses for the assignment are readily made. However, this also means that only moderate changes result in many regions of the IR spectrum due to the chemical transformation. Identifying the significant ones and isolating them from solvent signals can then become quite challenging. The electronic bands do change quite largely on the contrary, both in position and in strength. The problem there is the large homogenous and inhomogeneous width of each electronic transition. Sometimes even the decision of what is a separate electronic band or just a vibronic structure can become difficult.

Many of the seminal experiments in femtochemistry were done with fixed frequency pulses ${ }^{1}$ and this - in retrospect - required a fair amount of chemical intuition for the analysis. Broadband transient measurements have, however, been developed continuously and the change from liquid based continuum generation to crystal based continua has allowed an ever widening application. Due to the intrinsically broad spectrum of a femtosecond IR pulse - relative to the typical $15 \mathrm{~cm}^{-1}$ width of vibrational transitions - somewhat of an overview was originally easier to gain in this spectral range. ${ }^{2}$ The coverage of a wide spectral range requires tuning the probe pulse repeatedly and performing the time scan at each setting. This is where UV/vis transient spectroscopy has an intrinsic advantage, as extremely broad continua are now available ${ }^{3,4}$ and can be combined with detectors of a thousand or more pixels.

In this contribution we want to demonstrate the potential of a cutting edge broadband UV/vis/NIR transient spectrometer that will be briefly described in Sec. 2. We then present and discuss measurements on four sample systems ranging from ultrafast nonradiative decay, excited state proton transfer, and bond cleavage to ultrafast intersystem crossing. For all systems we find surprising new details that had been overlooked by the earlier investigations with limited spectral coverage. We thus can demonstrate that transient electronic spectroscopy is now becoming extremely powerful and the redundancy of information obtained in the measurements gives an unambiguous and clear picture of the complex dynamics. Combined with high level computations the energy flow in molecules after absorption of a photon and leading eventually to photochemistry can be traced most reliably. 


\section{Ultra-broadband transient spectroscopy with 50 fs resolution}

The transient spectrometer used for our measurements relies on a fully tunable pump source and a broadband continuum for wavelength selective probing. The general setup has been described in detail ${ }^{4}$ and is based on a $\mathrm{kHz}$ Ti:sapphire amplifier (CPA2001; Clark-MXR, Inc.). The pump pulses are derived from a blue pumped noncollinear optical parametric amplifier (NOPA) frequency doubled in a BBO crystal with typically $100 \mu \mathrm{m}$ thickness. The visible NOPA pulses are overcompressed in a fused silica prism compressor to precompensate for all dispersing elements after the doubling stage. With various mixing strategies and a possible intermediate continuum stage the range from $189 \mathrm{~nm}$ to $5 \mu \mathrm{m}$ can be covered without any gaps. ${ }^{5,6}$ The pump pulse lengths can be adjusted to $30 \mathrm{fs}$ or below from the deep UV to $1200 \mathrm{~nm}$ and are only slightly longer at longer wavelengths.

In the original setup we used a $775 \mathrm{~nm}$ pumped single filament supercontinuum that spans from 290 to $720 \mathrm{~nm}$ as the probe. Such a wide continuum is generated in a selected $5 \mathrm{~mm} \mathrm{CaF}_{2}$ plate that is translated in a circular fashion to avoid optically induced damage. The plate is carefully oriented to avoid depolarization of the continuum. ${ }^{7,8}$ The broadband probe light is dispersed in a fused silica prism and detected with a multichannel detector. The continuum chirp is corrected in the data postprocessing. The pump-probe delay up to $2 \mathrm{~ns}$ is adjusted with the help of a computer controlled delay line accurate to better than $10 \mathrm{fs}$. The overall temporal resolution is as good as $50 \mathrm{fs}$, mainly limited by residual group velocity mismatch between pump and probe in the $120 \mu \mathrm{m}$ solution sample. Transient spectrometers with parameters similar or close to ours are in use in a number of laboratories around the world. ${ }^{9-12}$

To access an even wider probe spectral range we alternatively pump the $\mathrm{CaF}_{2}$ based continuum generation by the frequency doubled or tripled Ti:sapphire
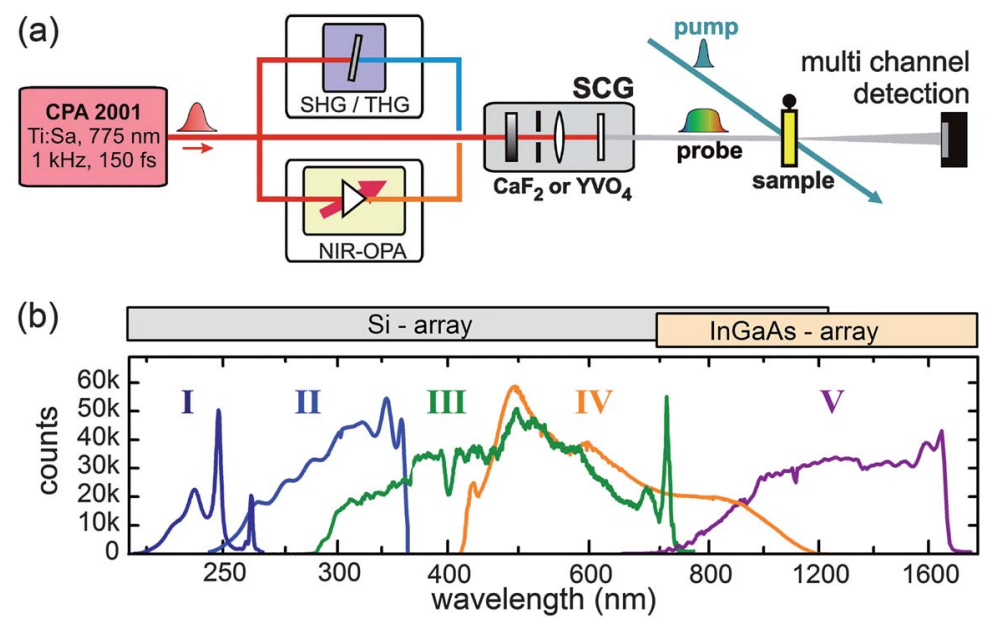

Fig. 1 (a) Scheme of the supercontinuum generation with various harmonics of the Ti:sapphire amplifier or the output of a NIR OPA. (b) Spectra of the continua as seen on the spectroscopic multichannel detector. 
output (see Fig. 1(a)). We find that significantly less pump energy is needed at these shorter wavelengths ( 387 and $258 \mathrm{~nm}$ ) and therefore no damage is found even in prolonged operation. The resulting spectra are shown in Fig. 1(b). They span from close to the pump wavelengths down to 245 and $225 \mathrm{~nm}$. The long wavelength cut-off is given by the dielectric filter used to suppress the high spectral power near the pump wavelength. Optimized filters with a smooth roll off could add some more spectral range in this vicinity to the probe pulse.

To access the near infrared (NIR) probe range we use a single-stage collinear optical parametric amplifier (OPA) to generate a red shifted continuum. ${ }^{3}$ With the OPA signal at $1200 \mathrm{~nm}$ and the $\mathrm{CaF}_{2}$ plate the useful continuum spans from 420 to $1100 \mathrm{~nm}$. In this range the silicon based CCD or diode array can still be used. For the 750 to $1700 \mathrm{~nm}$ range we use the idler at $2100 \mathrm{~nm}$ to generate the continuum in a $4 \mathrm{~mm} \mathrm{YVO}_{4}$ crystal. As detector an InGaAs based 256 pixel detector (Series 2000CV2 camera with uncooled Hamamatsu G9203-256D detector; Entwicklungsbüro Stresing) was used.

All continua are single filament and can therefore be well collimated and focused into the sample cell. Their spectra are summarized in Fig. 1(b). They overlap sufficiently and in total span from $225 \mathrm{~nm}$ to $1.7 \mu \mathrm{m}$. Typically two or three of the ranges are needed to cover the region of interest for a particular molecule. They are measured sequentially and then stitched together in the postprocessing to render the comprehensive manifold of transient spectra for all desired delay times.

\section{Selection of sample molecules and processes}

The development of the transient spectrometer described above has been motivated by our various investigations of ultrafast molecular and chemical dynamics. These range from photophysical process, via excited state electron and proton transfer to complex chemical reaction sequences. For this discussion we have chosen four examples that serve to demonstrate the newly available capabilities from the application point of view.

The first decision that has to be made in an ultrafast molecular experiment is the choice of the pump wavelength, $\lambda_{\text {pump }}$. As we have ultrashort pulses at all possibly needed wavelengths to our disposal, we choose $\lambda_{\text {pump }}$ purely according to the energy level structure of the molecule. We strongly believe that this enhances the value of the results as we do not have to worry about some initial electronic relaxation that is inevitably connected to some vibrational excitation. As shortly discussed above, such a choice of pump wavelength is always possible with the existing nonlinear pulse generation schemes.

The choice of $\lambda_{\text {pump }}$ can be made quite readily by inspecting the linear absorption spectrum of the sample. The spectra of the four substances are shown in Fig. 2. For 2,3,4,5-tetramethyl-2-cyclopentenone (TMCPO) in cyclohexane there is an extremely weak $\mathrm{S}_{1} \leftarrow \mathrm{S}_{0}$ transition around $320 \mathrm{~nm}$ and a quite strong $\mathrm{S}_{2} \leftarrow \mathrm{S}_{0}$ transition below $250 \mathrm{~nm}$. We excite TMCPO at $228 \mathrm{~nm}$ to specifically investigate the relaxation from the $\mathrm{S}_{2}$ state. Diphenylmethylbromide $\left(\mathrm{Ph}_{2} \mathrm{CH}-\mathrm{Br}\right)$ can be excited through the weak benzoic absorption at $270 \mathrm{~nm}$. In 2-(2'-hydroxy-5'methylphenyl)-benzotriazole (commercial name TINUVIN-P) ultrafast excited state intramolecular proton transfer (ESIPT) can be triggered by $\pi-\pi^{*}$ absorption at $350 \mathrm{~nm}$. The cationic dye malachite green (4-[(4-dimethylaminophenyl)phenyl-methyl]- 


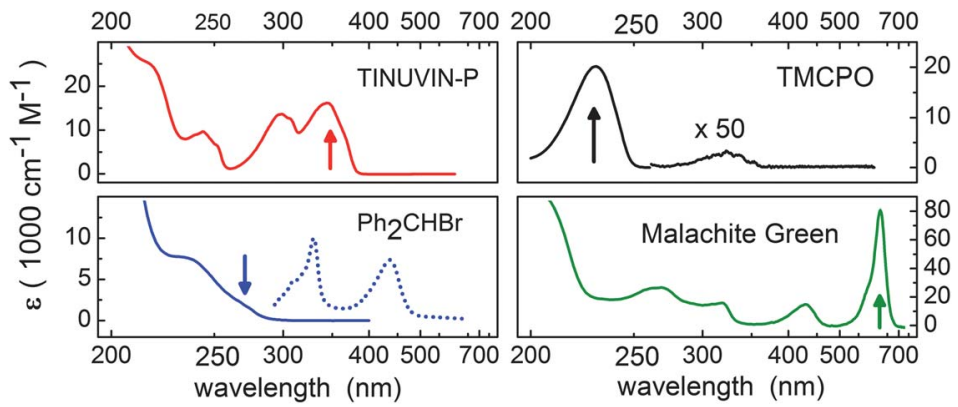

Fig. 2 Absorption spectra of the molecules studied in this work. TMCPO: 2,3,4,5-tetramethyl-2-cyclopentenone. In the panel for $\mathrm{Ph}_{2} \mathrm{CHBr}$ also the spectra of the benzhydryl radical and cation are shown (dotted line). The arrows indicate the pump wavelength.

$\mathrm{N}, \mathrm{N}$-dimethylaniline; $\mathrm{MG}$ ) is known to laser spectroscopists as it made the first real femtosecond laser system, the colliding pulse mode locked laser (CPM) possible due to its saturability at the $620 \mathrm{~nm}$ emission wavelength of Rhodamine 6G. In the present report we reinvestigate the ultrafast dynamics after 620 nm excitation.

\section{Putting the analysis of the ultrafast dynamics in malachite green on a sound basis}

Malachite green (MG, see Fig. 3(a)) is a cationic triarylmethane dye that absorbs strongly in the visible region. Since this absorption overlaps perfectly with the output of the CPM laser - it is actually used as a saturable absorber in this device it was one of the first molecules investigated for its ultrafast dynamics. The early studies concentrated on the ground state bleach (GSB) recovery. Rather complex kinetics with a main time constant of $2.1 \mathrm{ps}$ in methanol and a strong solvent dependence were found. ${ }^{\mathbf{1 3 , 1 4}}$ The solvent dependence is attributed to the viscosity of the solvent and it is postulated that an intermediate state $S_{x}$ is reached after
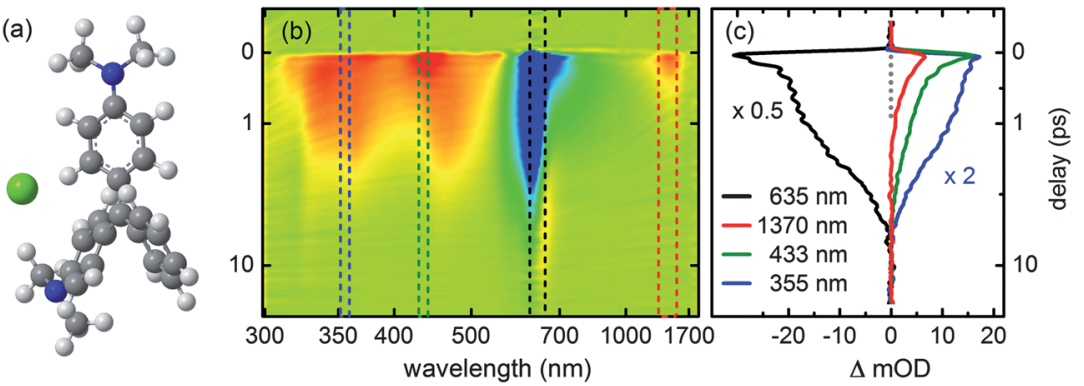

Fig. 3 Transient spectroscopy of malachite green dissolved in ethanol. (a) Structure of the dye. (b) Manifold of transient spectra. Red colours indicate an increase in absorption, blue colours a decrease. The wavelength scale is roughly reciprocal to pronounce different energetic parts equally. The time scale is linear in the range of -1 to +1 ps and logarithmic beyond. (c) Selected kinetic traces at the maximum of the NIR absorption of the LE state $(1420 \mathrm{~nm})$, the broad excited state absorption in the UV (350 $\mathrm{nm}$ ) and the GSB $(620 \mathrm{~nm})$. 
excitation through torsion of the phenyl rings. ${ }^{14-19}$ After relaxation from the $S_{x}$ state to the ground state the geometrical relaxation and cooling of the hot $S_{0}$ is thought to complete the cycle.

We investigated the dynamics of MG in ethanol after $620 \mathrm{~nm}$ pumping and utilizing the probe ranges III and V to cover the range from 300 to $1700 \mathrm{~nm}$. The resulting manifold of the transient spectra is shown in Fig. 3(b). A pump-probe cross correlation of $35 \mathrm{fs}$ in the NIR and slightly longer in the UV is reached and matches the resolution of previous one- ${ }^{\mathbf{1 4 , 2 0}}$ or two-colour pump-probe ${ }^{15}$ and fluorescence up-conversion ${ }^{18}$ measurements.

The strongest feature in the transient spectrum is the region of negative optical density around $635 \mathrm{~nm}$ (blue colours; Fig. 3(b))), where GSB and stimulated emission (SE) overlap. This is also the spectral region where most of the investigations were performed. In addition, the whole range from $300 \mathrm{~nm}$ to the GSB region and the NIR displays strong excited state absorption (ESA). A preliminary global fit analysis shows a 100 fs component with an increase of signal in the SE region and a decrease in the GSB region. This can be interpreted as the inertial motion of the optically prepared wavepacket away from the Franck-Condon point towards the equilibrium geometry of the excited electronic state $S_{1}$ that leads to a time dependent red shift of the SE. Such an extremely fast dynamics was already reported previously. ${ }^{14,20}$

A second decay component of about 440 fs has major peaks in the decay associated spectrum (DADS) at $433 \mathrm{~nm}$ (with $33 \mathrm{~nm}$ width) and at $1400 \mathrm{~nm}$ (430 $\mathrm{nm}$ width). These peaks can be readily assigned to the ESA following $\mathrm{S}_{1}$ excitation and leading to the $\mathrm{S}_{2}$ and $\mathrm{S}_{4}$ excited electronic states. In the absorption spectrum, transitions to these two states are found at the matching positions of 428 and $255 \mathrm{~nm}$ (compared in Table 1). A third spectral feature with a positive amplitude in the DADS is found to be nearly coinciding with the $\mathrm{S}_{1} \leftarrow \mathrm{S}_{0}$ absorption band, but is significantly different in shape. If this feature was due to a change in the GSB, it would indicate an increase in GSB with time. There is no argument known to us that would predict such a change in the hundreds of fs range. Therefore we have to assign this feature to the $S_{3} \leftarrow S_{1}$ transient absorption (ESA). Finally a decrease in SE with a 440 fs time constant is found that reaches from $635 \mathrm{~nm}$ to $1100 \mathrm{~nm}$. This matches the spontaneous emission spectrum within the limitation that certainly the stimulated emission in the sub-ps range does not originate from a thermalized $\mathrm{S}_{1}$ vibronic distribution. In summary, this means that the strong transient signal around the usual $620 \mathrm{~nm}$ pump

Table 1 Absorption energies for the $S_{n} \leftarrow S_{0}$ and $S_{n} \leftarrow S_{1}$ transitions obtained from the cw spectrum of MG together with the $S_{n} \leftarrow S_{1}$ transitions energies (widths in brackets) extracted from the transient absorption (TA) of MG. The time constants are obtained from single wavelength fits. " $d$ " signals where the time constant cannot be determined due to spectral overlap, "a" signals no amplitude for the associated time constant

\begin{tabular}{lllllll}
\hline & $\lambda(\mathrm{nm}) \mathrm{S}_{n} \leftarrow \mathrm{S}_{0}$ & $\lambda(\mathrm{nm}) \mathrm{S}_{n} \leftarrow \mathrm{S}_{1}$ & $\lambda(\mathrm{nm}) \mathrm{TA} \exp$ & $\tau_{1}(\mathrm{ps})$ & $\tau_{2}(\mathrm{ps})$ & $\tau_{3}(\mathrm{ps})$ \\
\hline $\mathrm{S}_{1}$ & 622 & & & 0.10 & 0.44 & 2.2 \\
$\mathrm{~S}_{2}$ & 428 & 1372 & $1400(430)$ & $\mathrm{a}$ & 0.44 & $\mathrm{a}$ \\
$\mathrm{S}_{3}$ & 315 & 638 & $\sim 635$ & $\mathrm{~d}$ & $\mathrm{~d}$ & $\mathrm{~d}$ \\
$\mathrm{~S}_{4}$ & 255 & 432 & $433(33)$ & $\mathrm{a}$ & 0.33 & 1.7 \\
ESA & & & 355 & $\mathrm{a}$ & $\mathrm{a}$ & 2.0
\end{tabular}


wavelength of most investigations is the sum of GSB, SE and ESA. It follows that the undiscussed averaging over the spectral width of the pump/probe pulse will not lead to any unambiguous interpretation of the dynamics of MG.

To display the transient absorption at selected times more clearly, we extracted the spectrum of absorption change (black lines in Fig. 4) from the complete manifold shown in Fig. 3(b). We then added a constant fraction of the ground state absorption spectrum (grey line in all panels of Fig. 4) to obtain the momentary spectrum (blue lines) of the sub-ensemble of molecules that was initially excited. The rapid decay of the 1400 and $433 \mathrm{~nm}$ peaks is clearly seen, as are the changes in the $635 \mathrm{~nm}$ region. At $1 \mathrm{ps}$, there is a broad absorption beyond the ground state absorption remaining that decays with about 2 ps. At 2 ps the 622 and $428 \mathrm{~nm}$ bands are the only remaining signals. The $622 \mathrm{~nm} \mathrm{~S}_{1} \leftarrow \mathrm{S}_{0}$ absorption band has, however, not yet fully recovered. It is interesting to notice that the shape of the $622 \mathrm{~nm}$ band does not significantly change during the relaxation processes. This is surprising in view of the generally accepted interpretation that large amplitude torsional motions of the phenyl rings dominate the dynamics. We cannot rationalize how such motions do not lead to changes in the $622 \mathrm{~nm}$ band of more than the $3 \%$ upper limit we can deduce as maximum spectral change compared to the full GSB signal.

Kinetic traces at selected wavelengths are shown in Fig. 3(c). The fitted exponential decay constants are shown in Table 1 . At $1400 \mathrm{~nm}$ we explicitly probe the $S_{1}$ population through the $S_{2} \leftarrow S_{1}$ ESA. We find a relaxation time of $440 \mathrm{fs}$ in good agreement with fluorescence measurements. ${ }^{21}$ It should be noted that the largely increased probe range of our experiment allows for this unambiguous determination of the $S_{1}$ lifetime by transient spectroscopy alone. At $433 \mathrm{~nm}$ there is an overlap between the specific $\mathrm{S}_{4} \leftarrow \mathrm{S}_{1}$ ESA and the very broad ESA that lasts into the ps range. The decay curve can be fitted with a first 330 fs exponential contribution that again signals the $S_{1}$ decay and a further 1.7 ps components. At $355 \mathrm{~nm}$ there is no ground state absorption and we do not have to expect any GSB. At this wavelength we can therefore exclusively probe the intermediate state $S_{x}$ that the $S_{1}$ state relaxes to with the $440 \mathrm{fs}$ time constant. The 2.0 ps decay is within experimental accuracy identical to the $1.7 \mathrm{ps}$ component already found at $432 \mathrm{~nm}$.

For the GSB recovery we need a $100 \mathrm{fs}$, a $440 \mathrm{fs}$ and a $2.2 \mathrm{ps}$ time constant. The first two most likely just result from the overlapping SE and ESA components. The

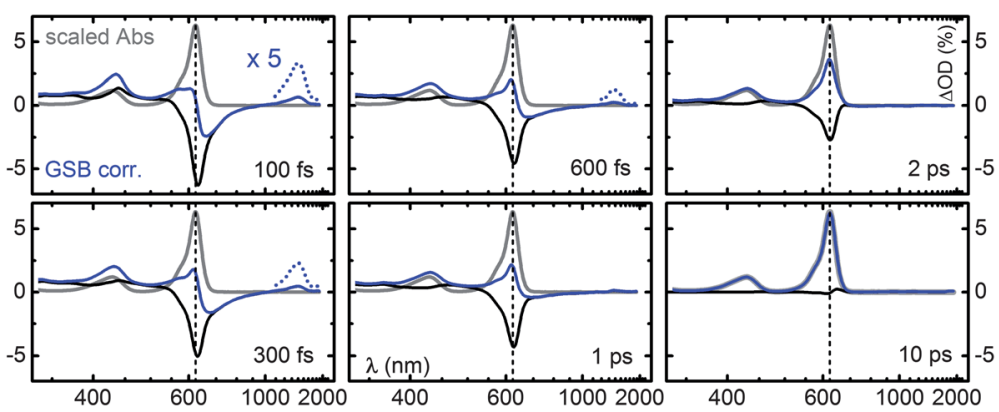

Fig. 4 Selected transient spectra at the indicated pump-probe delays (black lines). For visualization of the underlying changes in malachite green dissolved in ethanol, an equal amount of absorption spectrum is added to each transient spectrum (blue lines). In this way the recovery to the absorption spectrum (grey line) of the sub-ensemble excited by the pump pulse can be recognized. 
slightly longer 2.2 ps recovery time of the GSB as compared to the $\mathrm{S}_{x}$ decay is quite reasonable in view of cooling processes. Temporal signal oscillations of about $226 \mathrm{~cm}^{-1}$ that can be attributed to a symmetric carbon-phenyl stretch vibration ${ }^{14,22,23}$ are only found in the region from 605 to $635 \mathrm{~nm}$. This must mean that around state wavepacket prepared by resonant stimulated Raman scattering is observed.

The extremely wide probe range of our measurements and the detailed analysis leads to the following conclusions. After $S_{1}$ excitation MG relaxes to a state $S_{x}$ that neither fluoresces below $900 \mathrm{~nm}$ nor carries any oscillator strength to the known higher electronic states. Instead a nearly $2 \mathrm{eV}$ broad ESA band decaying with a time constant very close to the GSB recovery is found. This all points to a charge transfer (CT) character of the $S_{x}$ state. Indeed, recent high level calculations predict such a character already for the $S_{1}$ state ${ }^{24}$ and for the related molecule crystal violet a relaxation mechanism involving charge redistribution has been proposed..$^{25,26}$ We suggest that the initially excited (local excited) state has an electronic character more similar to the ground state. This property would explain the very strong $S_{1} \leftarrow S_{0}$ absorption. The often discussed torsion of the phenyl rings starts after the initial 100 fs geometric relaxation and leads to a simultaneous change in the electronic character towards a CT state on the half-ps time scale. Charge recombination then takes about 2 ps to repopulate the $S_{0}$ state with little optical signature from the "reverse" torsion of the phenyl rings.

From an extensive search of the literature it does not become clear, why repeatedly claims were made that the polarity of the solvent does not influence the dynamics of MG. It was always the viscosity that has been considered. If one, however, considers a series of alcohols with increasing size, not only the viscosity increases but also the polarity decreases. Future work should investigate this point by the reanalysis of existing data and solvent dependent transient spectroscopy. The solvation times of alcohols that are much longer than the ps times in the decay of excited MG are no intrinsic hindrance, as already a partial solvation can lead to efficient $\mathrm{CT}^{27}$ Given the capabilities demonstrated in this section, a final decision on the MG relaxation mechanisms should readily be possible.

\section{An overlooked twisting isomerization in the proton transfer cycle of TINUVIN-P}

TINUVIN-P is one of the best studied ESIPT systems. ${ }^{28-31}$ After a $60-80$ fs proton transfer the keto-type molecule relaxes to the electronic ground state with a 120-150 fs time constant. In the excited keto state a pronounced wavepacket motion is observed. ${ }^{31}$ As the internal conversion (IC) was assumed to happen in the closed keto form originating from the optically excited enol form, the subsequent 600 fs decay found at probe wavelengths in the visible region was assigned to the proton back-transfer..$^{29,30}$ In the enol form a $180^{\circ}$ twist around the central bond between the phenyl and the benzotriazole ring regenerates an identical conformation and a possible "isomerization" cannot be detected spectroscopically. In the keto-type form the same twist transfers TINUVIN-P from the closed form with the intramolecular hydrogen bond to the open form. ${ }^{32}$

The ultrafast proton transfer cycle leaves the TINUVIN-P in a highly vibrationally excited state. This is seen as a positive transient absorption below the $\mathrm{S}_{1} \leftarrow \mathrm{S}_{0}$ absorption edge at $367 \mathrm{~nm}$ and a decrease in absorption at shorter 

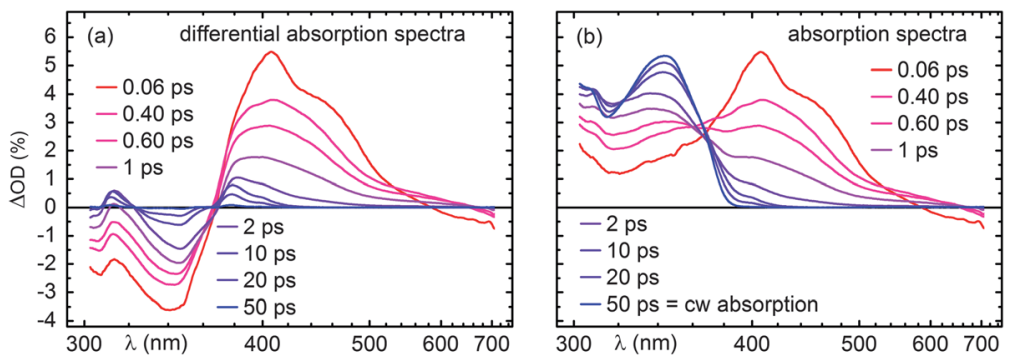

Fig. 5 (a) Transient absorption changes at selected delay times for TINUVIN-P in cyclohexane excited at $355 \mathrm{~nm}$. (b) Absorption spectrum of the sub-ensemble excited by the pump pulse.

wavelengths. ${ }^{33,34}$ For times from 5 ps onward the relaxation of this transmission change can be well modelled with a cooling process. However, it was already realized in the earlier investigations that the cooling picture was not sufficient to explain the spectral evolution in the first few picoseconds.

With our broadband spectrometer we investigated the dynamics of TINUVIN-P in cyclohexane. The differential absorption spectra shown in Fig. 5(a) display a rich variety of kinetics in the first 50 ps. To allow for an easier discussion of the underlying dynamics, we added a suitable fixed fraction of the ground state spectrum to all traces. The resulting time dependent absorption spectrum of the sub-ensemble excited by the pump pulse (see Fig. 5(b)) is used to discuss the observations.

At $60 \mathrm{fs}$ after the excitation depletion of the ground state absorption is clearly seen below $367 \mathrm{~nm}$. In the blue spectral region a strong transient absorption is found that initially displays some vibronic structure. This initial absorption must be due to ESA from the enol- or keto excited state. The absorption extends into the UV and overlaps with the ground state absorption. A weaker structure between 500 and $600 \mathrm{~nm}$ that develops with some delay is assigned to the closed keto ground state in accordance with ref. 30 . Beyond $600 \mathrm{~nm}$ stimulated emission is found. The SE decays very rapidly and therefore can be assigned to the excited keto-type state.

Kinetic traces at selected wavelengths are shown in Fig. 6. For clarity the traces are shown on an expanded scale in part (a,c) and over the full relevant range of 30 ps in part (b,d). At $684 \mathrm{~nm}$ we find a delay/increase of the SE with about 80 fs and a decay with $154 \mathrm{fs}$. Within the experimental precision this ESIPT and IC behaviour matches the previous reports. ${ }^{29,30}$ At $551 \mathrm{~nm}$ we find a $144 \mathrm{fs}$ increase of the signal and a 588 fs decay. Therefore we can confirm that in the 500 to $600 \mathrm{~nm}$ region the suggested proton back-transfer is observed and takes $600 \mathrm{fs}$.

At exactly the vibrationless $0-0$ transition of the optically pumped $\mathrm{S}_{1} \leftarrow \mathrm{S}_{0}$ band (367 nm) we find an isosbestic point in the sub-ensemble spectra (Fig. 5(b)) at times beyond some ps. This is in close agreement with the earlier reports ${ }^{33,34}$ and we can safely assign the decrease of absorption around $400 \mathrm{~nm}$ and the increase in the range around $350 \mathrm{~nm}$ to vibrational cooling. The spectral evolution of a cooling process does not render simple exponential decays with a wavelength independent decay time. Still, the value of $13 \mathrm{ps}$ found at $344 \mathrm{~nm}$ near the band maximum gives a characteristic time constant of the cooling process. A very detailed resonance Raman study finds mode specific behaviour with time constants in the same range. ${ }^{35,36}$ The traces at 362 and $375 \mathrm{~nm}$, energetically 


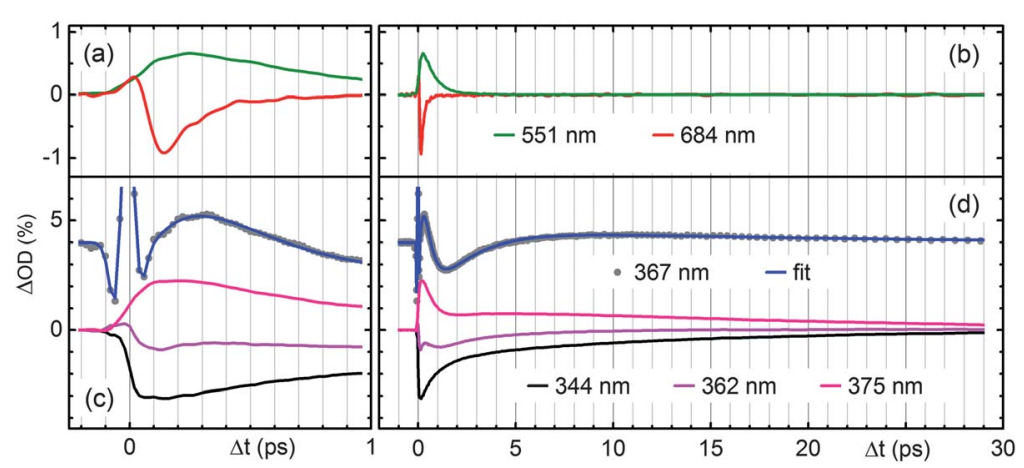

Fig. 6 Selected kinetic traces at the spectral positions, where the various conformers and relaxation processes of TINUVIN-P can be best differentiated. (a) and (c) show the traces within the first picosecond, while (b) and (d) show the range up to $30 \mathrm{ps}$. For clarity the coherent artefact due to the solvent cyclohexane has been subtracted from the traces except at $367 \mathrm{~nm}$. The trace for $367 \mathrm{~nm}$ is multiplied by a factor of 4 and shifted upward.

slightly above and below the $0-0$ transition consequently display quite complicated kinetics as many opposing effects contribute to the optical signal.

At exactly $367 \mathrm{~nm}$ the cooling does not influence the optical signal and the kinetics at this wavelength can therefore report the pure population evolution of the enol electronic ground state. We still have to assume that none of the other states involved in the full ESIPT and relaxation cycle contributes to the signal. Since the ESIPT itself proceeds in below $100 \mathrm{fs}$, the IC in $150 \mathrm{fs}$ and the backtransfer from the closed keto-type form in $600 \mathrm{fs}$, this is a fairly safe assumption after about 1 ps. Inspection of the $367 \mathrm{~nm}$ trace (Fig. 6(c,d); grey filled circles for the experimental values and blue line for a multi-exponential fit) shows indeed an $80 \mathrm{fs}$ decrease, a $150 \mathrm{fs}$ increase and a $410 \mathrm{fs}$ decrease. The vibrational cooling is seen as a very weak 15 ps decrease of the signal back to zero. Clearly there is another 2.2 ps contribution that was not seen in earlier work. In a global fit this time constant can be found as a prominent decay within most of the GSB range and the range around $450 \mathrm{~nm}$. However, single wavelength fits show a systematic variation of the time constant due to the cooling and the overlap with the ESA.

To assign this 2.2 ps relaxation constant we return to the red region of the spectrum. The width of the spontaneous emission spectrum of the closed ketotype form of TINUVIN-P is known ${ }^{31}$ and it is also established that the ground state closed keto-type form shows a mirror image to the fluorescence spectrum with a 0-0 transition at $625 \mathrm{~nm} .{ }^{30}$ Assuming a similar width and strength for emission and the absorption bands, only the region down to about $500 \mathrm{~nm}$ in the transient absorption spectrum can be assigned to the closed keto-type form. What is left to explain is the strong absorption in the range 380 to $500 \mathrm{~nm}$ and the $2.2 \mathrm{ps}$ time constant.

It has been calculated, that the IC of the excited closed keto-type form proceeds via a conical intersection that involves a twisting around the central bond and a pyramidization. ${ }^{32}$ A similar prediction backed up by experimental evidence was also made for the related 2-(2'-hydroxyphenyl)benzothiazole (HBT) ${ }^{37}$ As a result of the twisting motion necessary for the extremely fast IC, parts of the molecules do not relax to the closed keto-type form but to the open keto-type form. This is 
directly corroborated by transient IR spectroscopy ${ }^{38}$ and in line with early indications. ${ }^{29,39}$ In view of all the evidence we assign the 2.2 ps time constant in TINUVIN-P to the back-isomerization of the open to the closed keto-type form that is then followed by an even faster back-transfer of the proton to lead to vibrationally excited enol molecules. These are subsequently cooled by the solvent in 10 to 20 ps. The majority of the strong transient absorption in the blue is consequently assigned to the open form.

These additional species populated with the $150 \mathrm{fs}$ IC can readily explain the difficulties discussed previously to model the cooling kinetics at times before $5 \mathrm{ps.}^{34}$ It is not yet clear what this means for the interpretation of the resonance Raman studies. ${ }^{35,36}$ In summary, the relaxation cycle in UV-excited TINUVIN-P, that takes as little as some ten picoseconds for completion, involves forward ESIPT ( $\tau=80 \mathrm{fs}$ ), a branching in the IC to the closed and open keto-type form ( $\tau=150 \mathrm{fs})$, a back-twisting ( $\tau=2.2 \mathrm{ps})$, back-proton transfer $(\tau=600 \mathrm{fs})$ and finally cooling ( $\tau=15 \mathrm{ps}$ ). One might ask oneself whether such a complex sequence is a rare exception or a more general occurrence. The ring opening of 1,3-cyclohexadiene comes to mind with the subsequent multi-step relaxation to the lowest energy tZt-conformer. In this system also highly complicated kinetics were reported. ${ }^{\mathbf{4 0}}$

\section{The generation and interconversion of benzhydryl radicals and cations by photolysis of diphenylmethylbromide}

After UV excitation diarylmethylhalides undergo homolysis to the pair of a benzhydryl radical and halogen radical or heterolysis to the benzhydryl cation and the halogen anion. The overwhelming majority of investigations on this process and the subsequent interconversion between radical and ion pairs were performed without transient spectroscopy which renders spectra of the reactive intermediates, but rather by a priori chosen pump and probe pulse wavelengths. In addition, very intricate analysis with multiple exponential rates and a highly complex interpretation was deduced from these fixed colour recordings. ${ }^{41}$ Last but not least, only ps resolution was employed, even though it had been shown that the initial processes proceed on the sub-ps time scale. ${ }^{42}$

We utilized $30 \mathrm{fs}$ pulses at the first maximum of the absorption spectrum (270 nm; see Fig. 1) to excite either diphenylmethylchloride $\left(\mathrm{Ph}_{2} \mathrm{CH}-\mathrm{Cl}\right)$ or diphenylmethylbromide $\left(\mathrm{Ph}_{2} \mathrm{CH}-\mathrm{Br}\right)$. As the strong absorption features of the photolytic products $\mathrm{Ph}_{2} \mathrm{CH}^{+}$and $\mathrm{Ph}_{2} \mathrm{CH}^{-}$are well separated from the absorption of the precursors, we can use the range III of our continuum generation for a full process analysis. The spectrum of $\mathrm{Ph}_{2} \mathrm{CH}^{\circ}$ at $330 \mathrm{~nm}$ and the one of $\mathrm{Ph}_{2} \mathrm{CH}^{+}$at $430 \mathrm{~nm}$ are shown as dotted lines in Fig. 1.

The manifold of transient spectra for $\mathrm{Ph}_{2} \mathrm{CH}-\mathrm{Br}$ in acetonitrile at various pump probe delays is shown in Fig. 7(a). It can be readily seen that the radical signal appears very early after the optical excitation and decreases in the ps range. The cation signal increases more slowly and then also decreases later. The kinetic traces shown in Fig. 7(b) give a more detailed view. They show the integrated signal over the full bands. The optical signal can be considered to be proportional to the species population within good approximation. The step in the rise of $\mathrm{Ph}_{2} \mathrm{CH}^{\circ}$ (blue line) at about 50\% amplitude happens at $100 \mathrm{fs}$. In agreement with 

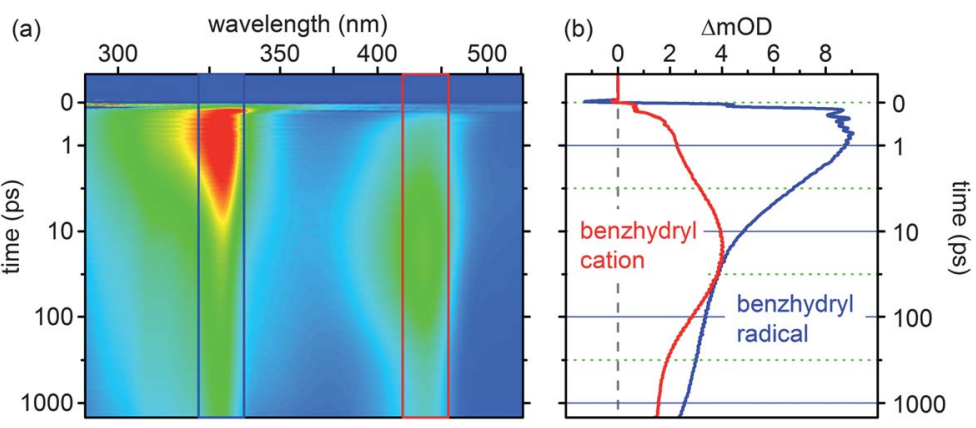

Fig. 7 (a) Transient spectra after $270 \mathrm{~nm}$ excitation of diphenylmethylbromide in acetonitrile. Red colour indicates a large transient absorption, green an intermediate one and blue colours little. (b) Signal for the resulting benzhydryl radical (blue line) and benzhydryl cation (red line) integrated over the respective band.

the observation for $\mathrm{Ph}_{2} \mathrm{CH}-\mathrm{Cl}$, where we observe such a step at $80 \mathrm{fs},{ }^{43}$ we assign this to the delayed generation of the radical. The excited state wavepacket propagates from the Franck-Condon region to a first conical intersection that leads part of the wavepacket to the radical product. The remainder of the wavepacket stays on the excited potential hypersurface and reaches a second conical intersection leading to the cation product. This takes a total time of $125 \mathrm{fs}$ in $\mathrm{Ph}_{2} \mathrm{CH}-\mathrm{Cl}$ and $200 \mathrm{fs}$ in $\mathrm{Ph}_{2} \mathrm{CH}-\mathrm{Br}$ (see step in the red line). This means that the cation does not appear until this later time. No more radical and cation population is generated at later times. The slight increase in the optical signals is rather due to an increase in transition strength due to solvation and planarization of the products. $^{\mathbf{4 4}}$

A close inspection of the product signal curves and the full set of transient spectra show significant modulations up to about $2 \mathrm{ps}$. The frequency is about 300 $\mathrm{cm}^{-1}$ for the radical and $350 \mathrm{~cm}^{-1}$ for the cation. To analyze these modulations, we compare the signal at the blue side of the radical absorption to that the red side (see Fig. 8(a)). Clearly a $\pi$ phase shift is seen that proves that the modulation is due to a vibronic wavepacket. In addition, we determined the position of the maximum of the radical and cation absorption for each delay. ${ }^{45,46}$ The resulting temporal dependence of the peak position shows the already mentioned ps relaxation due to solvation and planarization and also the oscillations. This confirms the interpretation as a vibronic wavepacket. An analysis of the normal modes of the radical and cation suggests that these wavepackets correspond to a symmetric stretch motion of the phenyl rings relative to the central carbon. This is very similar to the situation in MG (see above). However, in the photolysis of $\mathrm{Ph}_{2} \mathrm{CH}-\mathrm{Br}$ the wavepacket motion is in the photochemical products. The bond cleavage proceeds so fast that an impulsive excitation of the vibrational motion occurs. The stabilization of the radical and the cation leads to an increased electron density in the vicinity of the central carbon. Such an increase in electron density leads in turn to an impulsive shortening of the bonds and the suggested vibrational motion.

In the time up to about 10 ps the radical population decreases while the population of the cation increases. This is due to electron transfer between closely spaced benzhydryl and bromine radicals leading to the ion pair. We find that a 


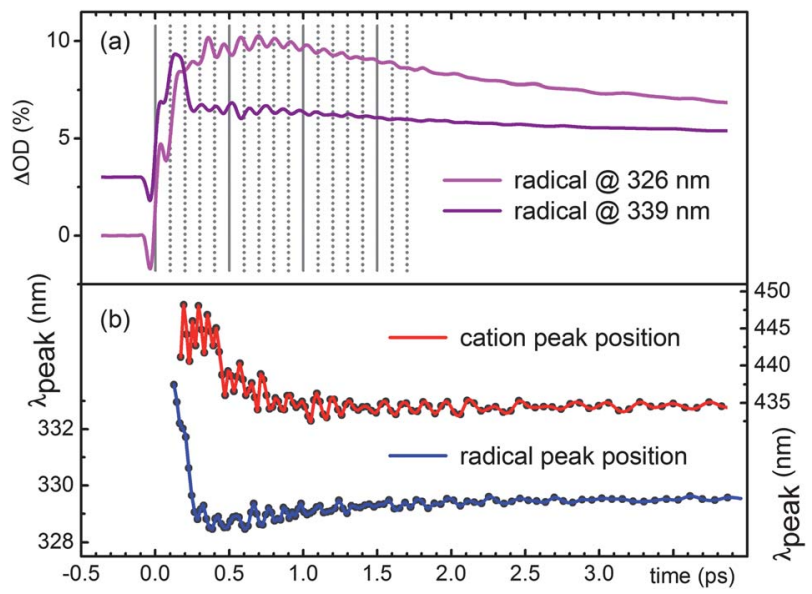

Fig. 8 (a) Transient absorption changes after $270 \mathrm{~nm}$ excitation of $\mathrm{Ph}_{2} \mathrm{CH}-\mathrm{Br}$ on the low wavelength $(326 \mathrm{~nm})$ and high wavelength $(339 \mathrm{~nm}$ ) edge of the radical product band. (b) Temporal dependence of the peak position of the benzhydryl radical (blue curve) and cation (red curve) band.

stretched exponential describes this process best, just as in our recent analysis of $\mathrm{Ph}_{2} \mathrm{CH}-\mathrm{Cl} .{ }^{46}$ The only difference is that for the chloride system an 11 ps time constant was found for this process compared to the shorter time constant of 2.1 ps in the bromide system. The decay of the radical population and the increase of the cation population with the identical temporal behaviour is direct proof of the interconversion by electron transfer. This finding is in contrast to the conclusions drawn from ps experiments on $\mathrm{Ph}_{2} \mathrm{CH}-\mathrm{Br}^{.47,48}$ Clearly the very fast termination of the electron transfer in the bromide system did not allow the correct interpretation in the earlier experiments with 20 ps resolution. The electron transfer is stopped by the depletion of closely spaced radical pairs. ${ }^{46}$

After the end of the electron transfer the remaining radical pairs separate by diffusion to give a stable population of free radicals. The ion pairs stick together for a longer time due to the Coulomb attraction. Therefore they can effectively undergo recombination and the reduced population of free ions eventually evolves (94 ps time for $\mathrm{Ph}_{2} \mathrm{CH}-\mathrm{Br}$ and 138 ps for $\mathrm{Ph}_{2} \mathrm{CH}-\mathrm{Cl}$ ).

The complete set of transient data cannot be modelled anymore with the usual kinetic scheme of well defined species like contact pairs, solvent separated pairs and free species. Instead we developed a combined model of Marcus-type electron transfer and Smoluchowski-diffusion. ${ }^{46}$ We have to assume an initial radical and cation pair distance distribution that extends far beyond the contact distance considered explicitly in earlier work. ${ }^{\mathbf{4 1 , 4 2 , 4 7 , 4 8}}$ The various processes are treated as distance dependent. The time dependent populations can then be deduced by integration over the full radial distribution for each delay time. The time dependent rates result from the convolution of the distributions with the distance dependent rates. ${ }^{46}$

The observation of the full range of optical signals connected with the photolysis of diarylmethylhalides with 50 fs temporal resolution renders many hitherto covered details on the dynamics. Hypotheses that have been carried on for decades can now finally be scrutinized. The investigation of complex and multistep chemical process is now possible in a most direct fashion. Together 
with high level theoretical considerations the influence of conical intersections $\mathbf{~}^{\mathbf{9}}$ and the microscopic arrangement can be investigated quantitatively. Since the breadth of optical signals renders a high degree of redundancy and cross checks, the ambiguity of many theories will be put to a serious test.

\section{Direct determination of the triplet yield after $S_{2}$ excitation}

One of the little mentioned understandings in ultrafast molecular dynamics seems to be that the rates connecting the originally excited state to the series of product states or chemical species are evaluated, presented and discussed, while the efficiencies and possible branching ratios are much less often put into the focus of the investigation. This stems from the fact that the yields of transient species are much less direct to determine compared to final product yields. For a hypothetical photochemical process leading from a precursor A to a stable product $\mathrm{B}$ the yield can readily be determined, as well established chemical means can be used to determine the product quantum yield. With modern illumination sources like high power LEDs this is extremely simple. ${ }^{50}$ As a by-product one finds out which fraction returns to the reactant and effectively just undergoes a photophysical nonradiative relaxation.

For transient species or states the situation is much more difficult. Even if a time resolved measurement is available and able to uniquely identify the transient species - as exemplified in the sections above - one can still not rely on the Beer-Lambert law as the molar absorption coefficient $\varepsilon$ of the species is generally not known. So only the product of $\varepsilon$ and concentration can be determined. For the benzhydrylbromide photolysis discussed in Sec. 6 one can chemically stabilize the cation and actually measure the value of $\varepsilon$. If in addition the fluence of the pump pulses at the sample position and all other relevant geometric parameters are determined, quantitative transient spectroscopy results that actually determines the time dependent yield of all species absolutely. ${ }^{46}$

More frequently other methods have been employed to determine the yield of intermediate species, particularly triplet states. Triplets are of special interest to chemistry as they are long-lived compared to singlet states and therefore can allow for diffusion controlled photo-initiated bimolecular processes like the ones found in photocatalysis ${ }^{51}$ and photo-initiated organic synthesis. One method uses triplet-triplet energy transfer to a system with a known triplet absorption coefficient and the measurement of the resulting quencher triplet by spectral photometry can be used. However this procedure requires very careful calibration and a certain degree of assumptions. The oxygen production by triplet-sensitized photooxidation of 1,3-diphenylisobenzofuran is an example of actually producing a physically measurable specific product that should be proportional in amount to the amount of triplet molecules produced over a selected period. ${ }^{52}$ Another spectroscopic method that requires many assumptions and checks is the measurements of the ESR spectrum that specifically can report the triplet concentration..$^{53}$ To decouple the triplet yield and the triplet absorption cross section, Van Stryland and coworkers have introduced a very elaborate doublepump method that relies on the partial saturation of transitions. This approach still has limitations given mainly by the molecular energy level structure. ${ }^{54}$ The most direct method is the determination of the ground state bleach recovery. This 
method relies on the ability to separate the time scales of the decay of the initially excited singlet and the resulting triplet in the GSB signal. This has been demonstrated by various single colour probe transient absorption measurements in the visible region. ${ }^{55-57}$

A more general application of the GSB recovery has so far been hampered by two issues. First, many systems of interest have their first strong absorption far in the UV. Second, a measurement at just one GSB wavelength still leaves the uncertainty that a superimposed ESA falsifies the interpretation. Both issues can now be solved with our extended probing range reaching as low as $225 \mathrm{~nm}$. This is certainly sufficient to reach the strong absorption bands of almost any substance of chemical interest. By recording a full transient spectrum, the shape of the known ground state absorption spectrum can be used to unambiguously disentangle GSB and ESA or product contributions.

For an experimental demonstration we chose 2,3,4,5-tetramethyl-2-cyclopentenone (TMCPO) in cyclohexane. As seen from the absorption spectrum displayed in Fig. 2, there is a very weak $S_{1} \leftarrow S_{0}$ absorption around $320 \mathrm{~nm}$ and a strong $\mathrm{S}_{2} \leftarrow \mathrm{S}_{0}$ absorption centred at $229 \mathrm{~nm}$. The assignment of the spectrum and the qualitative picture of the dynamics are made in analogy to the closely related acrolein. ${ }^{58}$ We pump the $S_{2}$ state with $228 \mathrm{~nm}$ pulses of sub-60 fs duration. The transient spectra are measured with the three continuum ranges I-III (Fig. 1(b)). They display very broad structures (see Fig. 9(a)) and it becomes clear that such a wide probe range is needed for a clear interpretation. The spectrum at 0.15 ps pump-probe delay is dominated by the $S_{2}$ state through its ESA and possibly direct stimulated emission. The TMCPO relaxes to the $S_{1}$ state with a time constant of $0.25 \mathrm{ps}$, a quite reasonable time for an IC mediated by a conical intersection. Subsequently the molecule relaxes to the triplet manifold within 4 ps. This is quite fast and the underlying mechanisms are presently under study (compare the scheme in Fig. 9(b)).

To determine the $S_{1}$ and triplet yield, we evaluate kinetic traces at probe wavelengths that allow for a straightforward interpretation. A full analysis shows that a kinetic scheme purely based on population transfer cannot describe the observed dynamics. A more complex model incorporating solvation and vibrational relaxation has to be considered. At $400 \mathrm{~nm}$ we can see predominantly the $\mathrm{S}_{2}$
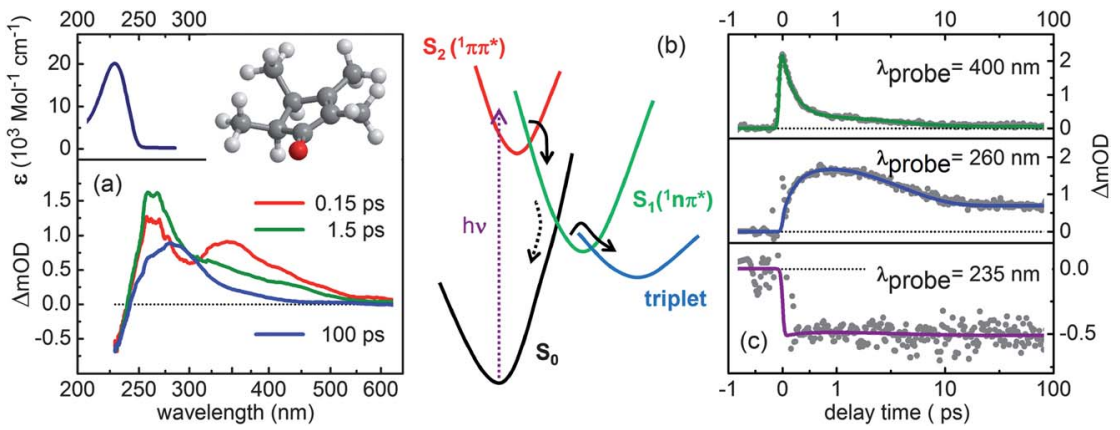

Fig. 9 (a) Selected transient spectra after $227 \mathrm{~nm}$ excitation of TMCPO in cyclohexane. The spectra were recorded in three parts and stitched afterwards. (b) Reaction scheme after $\mathrm{S}_{2}$ excitation. (c) Selected kinetic traces monitoring mainly the $S_{2}$ population $(400 \mathrm{~nm})$, the $S_{1}$ population $(260 \mathrm{~nm})$ and the GSB $(235 \mathrm{~nm})$. 
state relaxation with a $0.25 \mathrm{ps}$ exponential decay. At $260 \mathrm{~nm}$ the signal reports the $\mathrm{S}_{1}$ build-up and decay with 0.25 and $4 \mathrm{ps}$. Finally, at $235 \mathrm{~nm}$ the lack of any GSB recovery can be directly seen. Within the present experimental uncertainty given by signal fluctuations and the coherent contributions from the solvent and cell windows, we see no recovery of the GSB. This means that the whole $\mathrm{S}_{2}$ population initially generated by optical excitation transfers to the triplet. We can compare this to the previously reported triplet yield of the parent molecule cyclopentenone of also near unity. ${ }^{59}$ While an ultrafast intersystem crossing (ISC) proceeding with such a high yield is not unreasonable when the vibrationless $S_{1}$ state is excited, the finding of a unity yield after $\mathrm{S}_{2}$ excitation is surprising. The vibrational excess energy released in the $S_{2} \rightarrow S_{1}$ relaxation would easily suffice for IC to the electronic ground state as reported for acrolein. ${ }^{58}$ High level quantum chemical calculations do indeed show a low lying conical intersection between the $\mathrm{S}_{1}$ and the $S_{0}$ state. The fact that this process is highly inefficient while the ISC is highly efficient indicates that kinetic factors determine the dynamics and the branching ratio. That the triplet is accessed with near unity yield can be deduced from the transient spectra without any necessary assumptions as the GSB acts as an easily interpretable internal standard. The measurement is also corroborated by further recordings of TMCPO and 3-methyl-2-cyclopentenone in acetonitrile that also show near unity triplet quantum yield after $\mathrm{S}_{2}$ excitation.

\section{Summary and conclusions}

The four molecules investigated in this work all display complex multi-stage relaxation after optical excitation. This has in the past lead to investigations with partially contradicting interpretations and conclusions. The extremely broadband probe investigations now possible with about 50 fs temporal resolution render a detailed picture that allows an interpretation with unprecedented unambiguity. For each example a new twist in the story of the relaxation results that had been overlooked. In malachite green it appears that the importance of the phenyl twisting has been overemphasized and rather a charge transfer state should be considered. In TINUVIN-P the predicted twisting as the driving motion for the ultrafast IC is confirmed and leads to a resolution of the earlier puzzle that the sub-5 ps regime shows kinetics deviating from a pure cooling process. For the bond cleavage of $\mathrm{Ph}_{2} \mathrm{CH}-\mathrm{Cl}$ and $\mathrm{Ph}_{2} \mathrm{CH}-\mathrm{Br}$ the degree of electron transfer within the radical pair can now be determined quantitatively. The earlier single-wavelengths measurements with ps resolution lead to strongly contradicting conclusions. For the first time coherent wavepacket motion in the photoproducts is reported. Last but not least the measurement of the GSB recovery in the deep UV allows for the surprising result, that even after $S_{2}$ excitation of cyclopentenones the triplet states are reached with near unity probability within a few picoseconds.

All these investigations were purely based on UV/vis/NIR transient spectroscopy. As similar problems have been tackled in the past with transient IR spectroscopy, we would like to ask ourselves how the two methods with state-of-the-art setups, ${ }^{4,10-12,60-62}$ compare. For the comparison a figure of merit is helpful. A sensible figure of merit seems to be the ratio of the broadest spectral probe range available to the smallest width found in the spectrum. In the IR vibrational transitions in solution are about $15 \mathrm{~cm}^{-1}$ wide and the whole spectrum covers about $3000 \mathrm{~cm}^{-1}$. This gives a factor of 200 . The spectral width in the electronic 
spectrum is at least as wide as the homogeneous broadening determined by the pure electronic dephasing on the time scale of tens of fs. This converts to a linewidth of $200 \mathrm{~cm}^{-1}$. Our experimental setup can cover close to $40000 \mathrm{~cm}^{-1}$ probe range and again a factor of 200 results. This means that transient electronic spectroscopy has finally caught up with transient IR spectroscopy with respect to detection width.

In both kinds of spectroscopies one has to identify the relevant spectral regions where the intermediates can be most unambiguously identified and tracked. The continua used in our setup (compare Fig. 1(b)) cover huge ranges and together with the highly resolving UV/vis/NIR detector arrays a first overview can be gained from a single time scan measurement requiring one or two hours. The temporal resolution is typically 50 to $100 \mathrm{fs}$, somewhat superior to the IR. Most importantly we find that the extension to the NIR allows for a qualitative new feature. We can always probe to the red of the GSB and SE region where only ESA or product bands can be located. Identifying the "last transition" in the NIR then allows to obtain the kinetics of the single species. This is nicely seen in the MG transient peak at $1400 \mathrm{~nm}$ assigned to the $S_{2} \leftarrow S_{1}$ transition. Once the kinetics of this species are deduced from the measurement, the full associated spectral components can be extracted and used to decompose the set of transient spectra. In a second step the next to last transition is tackled and so on leading to a complete picture of photoinduced dynamics, however complex it might be.

The quantitative determination of transition strengths is readily possible by comparison with the intrinsic standard of the GSB of known strength. This is then often helpful to determine the electronic character of the intermediate species by comparison to quantum chemical calculations. What our setup also provides is sufficient spectral reproducibility and resolution to allow the observation of shifts as small as $0.1 \mathrm{~nm}$ or $10 \mathrm{~cm}^{-1}$. These can be identified with solvation, ion or radical pairing, ${ }^{46}$ and cooling. The temporal evolution of the shifts gives extra information on the dynamics.

It is also interesting to compare the ultra-broadband TA spectroscopy with fsfluorescence spectroscopy (fs-FS) as it can be measured with up-conversion ${ }^{63,64}$ or Kerr gating. ${ }^{65}$ The reason that much effort is put into developing techniques of fsFS is that the fluorescence is a very clean signature of the electronically excited molecule. There is no superposition in the signal by ESA or GSB as typically seen in TA spectroscopy with a limited detection range. As demonstrated in the examples of this work, ultra-broadband TA spectroscopy can fulfil the same condition by choosing the right spectral range, typically to the red of the GSB and stimulated emission. The well separated ESA transitions seen in this low energy part of the spectrum are also clean reporters of excited state dynamics. The great advantage is that the transient ESA signal can be measured with sub-50 fs temporal resolution, only reached by extremely well optimized fs-FS experiments. ${ }^{64}$ Contrary to fs-FS, where an improvement in temporal resolution leads to a largely decreased signal strength and long averaging times, the TA spectroscopy can be performed quite quickly. We therefore expect that particularly transient NIR detection will be able to rival fs-Fs without the need for a dedicated setup.

Last but not least we want to address the detection and identification of vibronic coherences. In many one-colour pump-probe measurements much debate is needed to find out whether the observed modulations in the TA signal are due to vibrational wavepackets in the electronic ground state or to vibronic 
wavepackets in an excited state. The first are a nice confirmation of our understanding of ultrafast experiments and the underlying molecular level structure, but they do not reveal much information beyond that already known from Raman spectroscopy and resonance Raman spectroscopy. Only the latter render real new information on the structure of the excited molecule and the course of reactions. We were able to report earlier that in ESIPT those modes contribute to the wavepacket signature that are intimately connected with the proton transfer by shortening of the donor-acceptor distance. ${ }^{66}$ Now we even find that in the bond cleavage discussed in Sec. 6 the photoproducts display long lasting vibrational coherences. This can only be understood if the whole cleavage process proceeds coherently. It gives great hope that coherent control, e.g., by double pulses or shaped pulses, can be used to control the branching ratio between homolysis and heterolysis and the yield of individual products. Also incoherent control by time delayed pulses of largely differing wavelengths might be feasible as the electron transfer, the recombination and the diffusional separation are understood in detail.

The newly available experimental capabilities should be able to revitalize the use of electronic spectroscopy for the elucidation and quantitative analysis of complex molecular and chemical processes. Unlike the typical use of UV/vis spectroscopy for stable species or just processes in the ms range, our strategy comes with 50 fs temporal resolution. As a consequence, even the shortest lived intermediates can be looked for. Unlike the earlier fs experiments the ultrabroadband detection allows the full range of spectroscopic knowledge to be utilized. We therefore think that the best is still to come for electronic spectroscopy and we look forward to a bright, visible future.

\section{Acknowledgements}

We thank P. Lang and Oliver Schalk for experimental help, and R. de Vivie-Riedle, S. Thallmair and T. Tahara for stimulating discussions. Financial support of this work by the Deutsche Forschungsgemeinschaft through the SFB 749, the GRK 1626, and the excellence cluster 'Munich Center for Advanced Photonics' (MAP) is gratefully acknowledged.

\section{References}

1 A. H. Zewail, Angew. Chem., Int. Ed., 2000, 39, 2586.

2 P. Hamm, S. Wiemann, M. Zurek and W. Zinth, Opt. Lett., 1994, 19, 1642.

3 M. Bradler, P. Baum and E. Riedle, Appl. Phys. B: Lasers Opt., 2009, 97, 561.

4 U. Megerle, I. Pugliesi, C. Schriever, C. F. Sailer and E. Riedle, Appl. Phys. B: Lasers Opt., 2009, 96, 215.

5 M. Bradler, C. Homann and E. Riedle, Opt. Lett., 2011, 36, 4212.

6 C. Homann, P. Lang and E. Riedle, J. Opt. Soc. Am. B, 2012, 29, 2765.

7 P. J. M. Johnson, V. I. Prokhorenko and R. J. D. Miller, Opt. Express, 2009, 17, 21488.

8 I. Buchvarov, A. Trifonov and T. Fiebig, Opt. Lett., 2007, 32, 1539.

9 R. Huber, T. Köhler, M. O. Lenz, E. Bamberg, R. Kalmbach, M. Engelhard and J. Wachtveitl, Biochemistry, 2005, 44, 1800.

10 A. N. Tarnovsky, W. Gawelda, M. Johnson, C. Bressler and M. Chergui, J. Phys. Chem. B, 2006, 110, 26497.

11 T. A. Zeidan, Q. Wang, T. Fiebig and F. D. Lewis, J. Am. Chem. Soc., 2007, 129, 9848.

12 A. L. Dobryakov, S. A. Kovalenko, A. Weigel, J. L. Pérez-Lustres, J. Lange, A. Müller and N. P. Ernsting, Rev. Sci. Instrum., 2010, 81, 113106.

13 E. P. Ippen, C. V. Shank and A. Bergman, Chem. Phys. Lett., 1976, 38, 611.

14 Y. Nagasawa, Y. Ando and T. Okada, Chem. Phys. Lett., 1999, 312, 161. 
15 Y. Nagasawa, Y. Ando, D. Kataoka, H. Matsuda, H. Miyasaka and T. Okada, J. Phys. Chem. A, 2002, 106, 2024.

16 D. Ben-Amotz and C. B. Harris, J. Chem. Phys., 1987, 86, 4856.

17 M. D. Hirsch and H. Mahr, Chem. Phys. Lett., 1979, 60, 299.

18 A. Mokhtari, L. Fini and J. Chesnoy, J. Chem. Phys., 1987, 87, 3429; A. Mokhtari, A. Chebira and J. Chesnoy, J. Opt. Soc. Am. B, 1990, 8, 1551.

19 F. Pellegrino, A. Dagen and R. R. Alfano, Chem. Phys., 1982, 67, 1982.

20 P. Sen, S. Yamaguchi and T. Tahara, Faraday Discuss., 2010, 145, 411.

21 N. H. Gottfried, B. Roither and P. O. J. Scherer, Opt. Commun., 1997, 143, 261.

22 M. J. Rosker, F. W. Wise and C. L. Tang, Phys. Rev. Lett., 1986, 57, 321.

23 K. A. Nelson and L. R. Williams, Phys. Rev. Lett., 1987, 58, 745.

24 A. Nakayama and T. Taketsugu, J. Phys. Chem. A, 2011, 115, 8808.

25 M. M. Martin, E. Breheret, F. Nesa and Y. H. Meyer, Chem. Phys., 1989, 130, 279.

26 Z. R. Grabowski, K. Rotkiewicz and W. Rettig, Chem. Rev., 2003, 103, 3899.

27 U. Schmidhammer, U. Megerle, S. Lochbrunner, E. Riedle and J. Karpiuk, J. Phys. Chem. $A, 2008,112,8487$.

28 S. R. Flom and P. F. Barbara, Chem. Phys. Lett., 1983, 94, 488.

29 M. Wiechmann, H. Port, W. Frey, F. Lärmer and T. Elsässer, J. Phys. Chem., 1991, 95, 1918.

30 C. Chudoba, S. Lutgen, T. Jentzsch, E. Riedle, M. Woerner and T. Elsaesser, Chem. Phys. Lett., 1995, 240, 35.

31 C. Chudoba, E. Riedle, M. Pfeiffer and T. Elsaesser, Chem. Phys. Lett., 1996, 263, 622.

32 A. L. Sobolewski, W. Domcke and C. Hättig, J. Phys. Chem. A, 2006, 110, 6301.

33 W. Frey and T. Elsaesser, Chem. Phys. Lett., 1992, 189, 565.

34 K. Lenz, M. Pfeiffer, A. Lau and T. Elsaesser, Chem. Phys. Lett., 1994, 229, 340.

35 V. Kozich and W. Werncke, J. Mol. Struct., 2005, 735, 145.

36 V. Kozich, J. Dreyer and W. Werncke, Chem. Phys. Lett., 2004, 399, 484.

37 M. Barbatti, A. J. A. Aquino, H. Lischka, C. Schriever, S. Lochbrunner and E. Riedle, Phys. Chem. Chem. Phys., 2009, 11, 664.

38 O. F. Mohammed, S. Luber, V. S. Batista and E. T. J. Nibbering, J. Phys. Chem. A, 2011, 115, 7550.

39 P. F. Barbara, L. E. Brus and P. M. Rentzepis, J. Am. Chem. Soc., 1980, 102, 5631.

40 S. Lochbrunner, W. Fuss, W. E. Schmid and K.-L. Kompa, J. Phys. Chem. A, 1998, 102, 9334.

41 K. S. Peters, Chem. Rev., 2007, 107, 859.

42 M. Lipson, A. A. Deniz and K. S. Peters, Chem. Phys. Lett., 1998, 288, 781.

43 C. F. Sailer, N. Krebs, B. P. Fingerhut, R. de Vivie-Riedle, E. Riedle, Phys. Rev. Lett., submitted for publication.

44 B. P. Fingerhut, C. F. Sailer, J. Ammer, E. Riedle and R. de Vivie-Riedle, J. Phys. Chem. A, 2012, 116, 11064.

45 C. F. Sailer, R. B. Singh, J. Ammer, E. Riedle and I. Pugliesi, Chem. Phys. Lett., 2011, 512, 60.

46 C. F. Sailer, B. P. Fingerhut, S. Thallmair, C. Nolte, J. Ammer, H. Mayr, I. Pugliesi, R. de Vivie-Riedle and E. Riedle, ChemPhysChem, 2013 (in print).

47 J. Dreyer and K. S. Peters, J. Phys. Chem., 1996, 100, 15156.

48 J. Dreyer, M. Lipson and K. S. Peters, J. Phys. Chem., 1996, 100, 15162.

49 B. P. Fingerhut, D. Geppert and R. de Vivie-Riedle, Chem. Phys., 2008, 343, 329.

50 U. Megerle, R. Lechner, B. König and E. Riedle, Photochem. Photobiol. Sci., 2010, 9, 1400.

51 M. Wenninger and E. Riedle, in Chemical Photocatalysis, ed. B. König, DeGryuter, Berlin, 2013.

52 M. R. Detty and P. B. Merkel, J. Am. Chem. Soc., 1990, 112, 3845.

53 M. Guéron, J. Eisinger and R. G. Shulman, Mol. Phys., 2006, 14, 116.

54 D. Peceli, S. Webster, D. A. Fishman, C. M. Cirloganu, H. Hu, O. V. Przhonska, V. V. Kurdyukov, Y. L. Slominsky, A. I. Tolmachev, A. D. Kachkovski, R. R. Dasari, S. Barlow, S. R. Marder, D. J. Hagan and E. W. Van Stryland, J. Phys. Chem. A, 2012, 116, 4833.

55 H. E. Lessing, A. von Jena and M. Reichert, Chem. Phys. Lett., 1976, 42, 218.

56 S. M. King, C. Rothe, D. Dai and A. P. Monkman, J. Chem. Phys., 2006, 124, 234903.

57 B. Lament, J. Karpiuk and J. Waluk, Photochem. Photobiol. Sci., 2003, 2, 267.

58 A. M. D. Lee, J. D. Coe, S. Ullrich, M.-L. Ho, S.-J. Lee, B.-M. Cheng, M. Z. Zgierski, I.-C. Chen, T. J. Martinez and A. Stolow, J. Phys. Chem. A, 2007, 111, 11948.

59 R. Bonneau, J. Am. Chem. Soc., 1980, 102, 3816.

60 E. T. J. Nibbering and T. Elsaesser, Chem. Rev., 2004, 104, 1887.

61 E. T. J. Nibbering, H. Fidder and E. Pines, Annu. Rev. Phys. Chem., 2005, 56, 337. 
62 M. L. Groot, L. J. G. W. van Wilderen and M. Di Donato, Photochem. Photobiol. Sci., 2007, 6, 501.

63 X.-X. Zhang, C. Würth, L. Zhao, U. Resch-Genger, N. P. Ernsting and M. Sajadi, Rev. Sci. Instrum., 2011, 82, 063108.

64 C. H. Kim and T. Joo, Opt. Express, 2008, 16, 20742.

65 B. Schmidt, S. Laimgruber, W. Zinth and P. Gilch, Appl. Phys. B: Lasers Opt., 2003, 76, 809. 66 S. Lochbrunner, A. J. Wurzer and E. Riedle, J. Phys. Chem. A, 2003, 107, 10580. 